\title{
Analysis of the Efficacy of Acupoint Sticking Therapy in the Treatment of Pediatric Bronchial Pneumonia after Anti-infection Criteria
}

\author{
Shuling Wang, Jianghong Hou, Tuanying Chen, Dongdong Shen
}

Henan Province Hospital of Traditional Chinese Medicine, Zhengzhou, Henan 450002

Keywords: acupoint sticking therapy; pediatric bronchial pneumonia

\begin{abstract}
Pneumonia is one of the most common diseases in pediatric patients. Some children with pneumonia have common problems such as difficulty in absorption of lung rales, unsatisfied disease, or poor compliance during clinical treatment. The application of acupoint is one of the external treatments of the motherland medicine and has shown its unique effects in the treatment of children with pneumonia. This paper reviews the literature on the application of acupoint in children with pneumonia in recent 10 years, and provides a basis for promoting the use of acupoint in the treatment of children with pneumonia.
\end{abstract}

\section{Introduction}

Pediatric pneumonia is a common clinical respiratory disease, and its incidence and mortality rate are the highest. It poses a great threat to the healthy growth and life safety of children. Children with pneumonia are usually accompanied by fever, cough, chest tightness, shortness of breath and other symptoms. As the disease progresses, cough, shortness of breath and aggravation, there will be dyspnea, nod breathing and other breathing difficulties, the previous clinical treatment of western medicine, However, due to the abuse of antibiotics caused by increased drug-resistant strains, simple Western medicine treatment is poor. With further clinical research, it has been found that Chinese medicine has a good prospect for the treatment of pediatric pneumonia. It not only has good curative effect, but also has little side effects, and can effectively enhance the immunity of children and reduce airway reaction. However, due to the bitter taste of Chinese herbal medicine, most of the children resisted oral administration, and poor compliance made the efficacy of Zhongtang medicine unable to be fully exerted. Therefore, acupoint application of traditional Chinese medicine was used for clinical treatment, and acupoint application was a new route of administration. Can effectively promote the absorption of inflammation and shorten the treatment time [1]. In view of this, this study further explores the clinical efficacy of Chinese medicine acupoint application in the treatment of children with pneumonia.

\section{The Theoretical Basis of Acupoint Application}

Acupoint application is one of the external methods of Chinese medicine. Its theoretical basis mainly stems from three aspects: (1) The overall concept. Chinese medicine believes that the human body is centered on the five internal organs and connects six meridians, five bodies, five features, nine skeletons, and four limbs and limbs through the meridian system into an organic whole, and then completes the body's functional activities through the functions of refinement, qi, blood, and body fluids. The "Lingshu • Sea Theory" cloud: "Five menstrual meridians who belong to internal organs, outside the network of limbs." (2) Meridian theory. The meridian is an important part of the human body's organizational structure. It is a channel that runs the body's qi and blood, communicates with organs and limbs, and communicates both inside and outside. "Lingshu • by ribs," said: "The meridians, so we must die, at all diseases, adjusting the actual situation, can not be unreasonable." (3) physiological function. Acupoints are special parts of the body's organs and meridians for blood transfusion. They have special physiological functions. Each acupoint has its uniqueness and bidirectional regulation. It is quite sensitive to drugs and can make the physicochemical action of the drug stay or release to the whole body for a long time to produce 
overall regulation [2]. Acupoint sticking therapy is based on the overall concept of traditional Chinese medicine, through the direct effect of drug absorption and stimulation of acupuncture points to stimulate the indirect effect of gas production to achieve the purpose of treatment. Modern researches have proved that drugs act on the acupoints of the human body from the outside of the human body. The acupoints of the skin, tissue structure, blood vessels, lymph, and nerves all undergo certain changes. Some drugs can stimulate acupuncture points, increase local temperature, and dilate capillaries. It facilitates penetration of the drug ingredients into the skin and into the lymph and blood to exert related pharmacological effects. Acupoint sticking therapy can also stimulate the absorption and metabolism of drugs by stimulating acupuncture points, have a certain influence on the body's related chemical and physical receptors, reflexly adjust the function of the autonomic nervous system, and enhance immunity through cell and humoral immunity. To achieve the purpose of disease prevention and treatment [3]. Children's delicate skin, good skin tissue permeability, and more conducive to drugs through the skin to stimulate acupuncture points, so children with acupoint application therapy is particularly prominent.

\section{Acupoint Selection}

In the literature on the treatment of pediatric pneumonia in related acupoints, the selection of acupoints is more flexible, including single-point treatment, multi-hole combination, fixed acupoint selection, and acupoint selection and differentiation. But most of them are mainly back acupoints. There are clouds in the "Li Shu Wen": "The five internal organs are salty and back, and the twelve organs are on the back. The acupuncture points can be evil, so the organs can all be cured." The application of back acupoint sticking therapy has the energy to stimulate the yang, Wentong Zhuquan, so that blood and blood flow smoothly, expelling evil to reach the table.

In the single-point treatment, Feishu, Ashi (the wet rales is the most obvious place) and Shenfu are the main ones, among which the single-choice Feishu is the most [4]. The Feishu point is the acupoint of the back of the bladder of the foot of the sun. It treats the lungs and respiratory diseases. It is the place where the lungs transfuse and transfuse. The medicine is applied here. It has the function of relieving the lungs, relieving the lungs, and relieving the lung. Respiratory disease-related symptoms improve lung function. The Shenque point belongs to the Renmai, and it is the root of the Tibetan spirit that belongs to the Governor. The medicines can be applied here through the appropriate organs to drive off the evils, warm the hernia, raise and lower the turbidity, and regulate the air conditioner. Ma Wenqiang et al selected Feishu points for treatment with pneumonia patch, compared with the use of Western medicine alone, the symptoms of children with better symptoms are better. Ge et al. used 40 children with pneumonia as an observation group, and added "Yi Sui San" to apply Shen Shen therapy on the basis of conventional treatment. The total effective rate was higher than that of the control group, and the difference was statistically significant $(\mathrm{P}<0.05) .<0.05)$

The selection of fixed acupuncture points was Feishu, Tiantuo, Jizhong, Dazhui, Dingchuan, Shenfu, and Gaozhi. In addition, there were still many combinations of Pishuyu, Yushu, Shenyu, Bailu, Zusanli, Yamen, Xinyu, Yongquan points and other acupuncture points. In the treatment of children with pneumonia, most of them are mainly from backshu acupoints and Renmai acupoints. Liu Xiuping [5] In a study of 50 cases of children with pneumonia, the treatment group was given a traditional Chinese medicine patch on the basis of conventional anti-inflammation and applied to the children Feishu, Jizhong and Dazhui. Feishu and Yuzhong acupoints are located in the middle of the two lungs. Dazhui is called Sanyang and Governor Vessels. Combined use of points can lead to improvement of pulmonary circulation and promotion of inflammatory absorption. Chen Yu [9] 80 cases of pneumonia were randomly divided into two groups, the treatment group selected Tianxu, Fuzhong, bilateral Feishu 4 points to observe the efficacy. The total effective rate of the treatment group was better than that of the control group. Tian Tuo is a Renmai. Shi Rong et al. [10] and Fu Shi In the treatment of children with pneumonia, they all selected the Zhaizhong, Feishu, Shenque and Tiantu points. The efficacy and clinical cure rate of the experimental group were better than those of the control group. 


\section{Clinical Data}

A total of 40 patients were from acupuncture clinics in our hospital from October 2012 to March 2014. 23 males and 17 females; aged 8 months to 9 years old, an average of 4.5 years; duration of 20 days to 6 months, an average of 1.5 months. After the treatment of anti-infective treatment is still repeated cough, sputum. Inclusion criteria: 1 Conforms to "Pediatric Disease Diagnosis and Treatment Criteria" Diagnostic Criteria for Pediatric Pneumonia, with reference to "Standards for the Diagnosis and Efficacy of TCM Syndrome Diseases". 2 have been diagnosed with pneumonia, and standard anti-infective treatment for at least 1 week. 3 With cough, expectoration as the main clinical symptoms. Exclusion criteria: 1 high fever did not retreat; 2 tonsil swelling greater than II degree; 3 point application of skin ulceration or infection; 4 and esophageal reflux, pharyngeal nasal drip leakage.

Drugs: Take white mustard seeds, clematis, ephedra, garcinia, asarum, almonds, honokiol, schisandra and pulverize in proportions of 2:5:3:5:2:3:1:1. Take some powdered ginger water and mix thoroughly. In the dough for spare. Acupoints: Feishu, body pillars, feet Ze, Tianxu, Yuzhong (any side of acupuncture points on either side). Severe cough accompanied by vomiting or phlegm and phlegm, pharyngeal itching plus Zhaohai, nasal congestion increased vertebral, pulmonary auscultation wet rales heavier plus bilateral Zhongfu points. Method: Place the prepared drug on the applicator and apply it according to the selected point. The first post about 40 minutes, if the tolerance is good, no local allergies and other adverse reactions, can gradually increase the application time, 1 day before the first 5 days, 5 days after the other day, every $40 \mathrm{~min} \sim 2 \mathrm{~h}$. After 10 days of treatment, the curative effect was evaluated. If the skin can not tolerate burns, it can be compound lithospermia oil, cool oil, aloe vera juice or scald cream Waicha. If blisters can be sterilized and then picked up, to avoid the original acupoint application.

\section{Typical Cases and Discussions}

Zhang, male, 2 years and 5 months, first diagnosed on September 28, 2013. One month ago, I got fever $39.5^{\circ} \mathrm{C}$ after getting cold, and I had cough and shortness of breath. I was hospitalized in our pediatric department. Leukocytes 15.6 x 109/L, neutrophils 0.79. Chest radiographs suggest multiple patches of scattered shadows in the lungs. Auscultation can be heard in both lungs and buzzing sounds and fixed wet rales. The hospital was discharged from the hospital 10 days after being treated for fever, anti-infection (intravenous ceftriaxone sodium), and atomization. After the cough still exists, the use of Huayu Xuanfei Chinese medicine oral 1 week oral efficacy is poor. Cough, phlegm aggravated at night and early morning, severe cough vomiting nausea, anorexia, normal bowel movements, disturbed sleep, fingertips slightly darker in the wind off, auscultation breath sounds thick lungs. The diagnosis was cough (phlegm blocking, pulmonary failure). To be applied to Tianxu, Jizhong, Zhongyu, Shizhu and Zhaze. On September 29, there was no nausea. After continuing to apply, there will be no more cough or phlegm after 5 days, and the appetite will be restored. Apply it on the body pillars, Ojo, and ointment every week to consolidate the curative effect. The basic pathogenesis of cough is the loss and decline of lung failure. Acupoint sticking therapy is the further development of Tianmo moxibustion. The prescription consists of mustard seed, clematis, ephedra, garcinia, asarum, almond, honokiol, and schisandra. Among them, mustard seeds, garcinia, etc. have the role of permeabilization. Asarum and ephedra oscillate divergently to restore the power of lung qi. Almonds and magnolias settled down to restore the lung's desiccation characteristics. Ephedra takes the lungs, and Magnolia enters into the large intestine to treat both internal and external. Clematis contains pulsatillae, pulsatilla and saponins, etc. It has antibacterial, anti-inflammatory and relaxing smooth muscle functions. It is a commonly used drug for the treatment of intractable cough. The compatibility of acupoints is mainly to restore the normal functions of the hand lunar lung meridians and lungs. Feishu has the function of adjusting organs, and it is the basic point for treating lung diseases. The body pillar has the effect of reducing the antitussive and regulating the spleen and stomach, and is a key point for children's health care [5]. Zeze is a combination of lungs and lungs, which has the effect of reducing the lung pressure. 
Jizhong is a "qi meeting" and has the effect of adjusting the air-conditioning of the air-conditioning system and restoring the air-in/out of the air-conditioning system. Tiantu points belong to the nearby points and strengthen cough relieving effect. Acupoint dressing therapy also has some deficiencies. For example, children have uncomfortable reactions to ginger dressings, such as stinging and red itching. Second, only the symptoms of coughing. Therefore, it is necessary to further optimize the program and design more rigorous clinical trials to further verify and popularize this external therapy that is easy to use, easy to use, and easy to use.

\section{Conclusion}

Acupoint application has broad prospects for the treatment of children with pneumonia. It is a treatment that combines meridians, acupoints and drugs. Therefore, a reasonable and comprehensive analysis of acupoints and drug selection, transdermal technology, TCM syndrome differentiation and meridian transmission will provide better results for the treatment of children with pneumonia.

\section{References}

[1] Xiao Dongwei, Gao Mingli. Discussion on theoretical basis of acupoint application[J]. Journal of Liaoning University of Traditional Chinese Medicine,2008,10(5):127.

[2] Zhang Yanhong. Theoretical basis and current application of acupoint application [J]. Western medicine, 2007, 20 (2): 1-3.

[3] Liu Weiai, Ye Debao. Research progress of transdermal drug delivery with acupoint patch therapy [J]. Chinese Medicine Bulletin, 2004, 3 (4):59-62.

[4] Qian Yuping. White mustard seeds for topical treatment of children with pneumonic rales malabsorption in 84 cases [J]. Journal of Henan University (Medical Science),2006,25 (1):60-61.

[5] Wang Jingbo, Wang Zuozhong, Wei Lanfang. Therapeutic effect of internal and external combination of Chinese and Western medicine on children with pneumonia and asthma (attack period) 60 cases [J]. New Chinese Medicine, 2010 (11): 33-34. 